\title{
Elargissement du réseau de nos sociétés fiduciaires partenaires dans le canton de Vaud
}

FMH Consulting Services présente un nouveau partenaire en matière fiduciaire à Lausanne.

Messieurs Patrick Pasquier et Gérard Dustour sont à votre disposition.

La société CRF Fiduciaire et Conseils S.A. dont le siège est à Nyon a été créée en 1988 par M. Pasquier; elle compte actuellement 14 personnes, M. Dustour I'a rejointe en 2002. CRF est membre de la Chambre fiduciaire, ce qui garantit une exécution des mandats selon les normes de la profession.

CRF est active dans tous les domaines fiduciaires, notamment:

- comptabilité

- fiscalité personnes physiques et morales

- études de financements

- conseils financiers

- assistance lors de la remise de cabinet

- ressources humaines et gestion des salaires

Ceci aussi bien pour des entreprises d'une certaine importance que pour des indépendants dont de nombreux médecins.

Nous sommes à même de fournir des prestations qualitatives avec un bon rapport qualité-prix. Nous pouvons mettre en exergue la stabilité du personnel de notre société, qui est un gage de continuité et d'un suivi personnalisé des mandats.

Nous disposons également d'un accès facile avec des places de parking pour nos clients.

Nous sommes à votre entière disposition et nous nous réjouissons de votre appel!

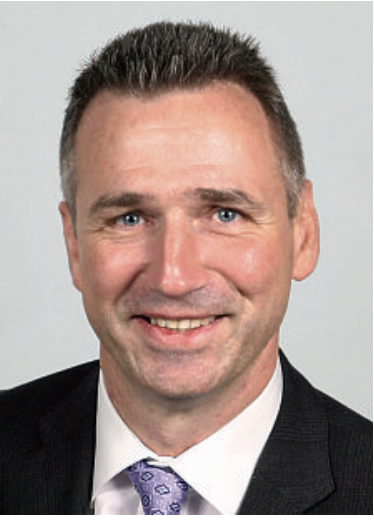

Patrick Pasquier

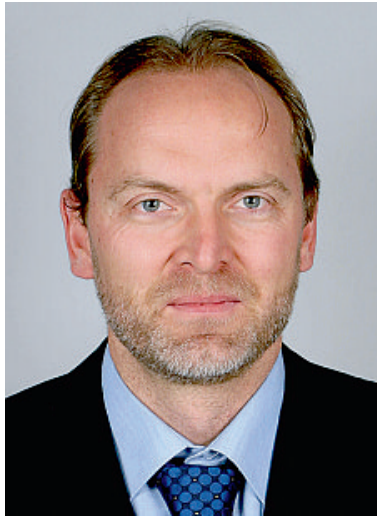

Gérard Dustour

\section{Siège \\ CRF Fiduciaire et Conseils S.A. \\ FMH Fiduciaire Services \\ $50 \mathrm{~A}$, route de Divonne \\ Case postale 2207 CH-1260 Nyon 2 \\ Tél. 0223655959 — Fax 0223655940 \\ patrick.pasquier@fmhfiduciaire.ch \\ gerard.dustour@fmhfiduciaire.ch \\ www.fmhfiduciaire.ch \\ Succursale \\ CRF Fiduciaire et Conseils S.A. \\ FMH Fiduciaire Services \\ 17, avenue Villamont $\square \mathrm{CH}-1005$ Lausanne \\ Tél. 0213235959 口ax 0213235956 \\ patrick.pasquier@fmhfiduciaire.ch \\ gerard.dustour@fmhfiduciaire.ch \\ www.fmhfiduciaire.ch}

\section{ĞFMH SERVICES}

\section{Nos prestations}

- Conseils pour l'ouverture et remise/reprise du cabinet

- Séminaires pour la gestion d'un cabinet médical

- Conseils en matière d'assurance

- Calcul de la valeur d'un cabinet

- Achats collectifs pour les médecins
- Encaissement d'honoraires

- Office de placement

- Planification et aménagement

- Offre spéciale - carte de crédit

- Conseils financiers/budget

FMH Consulting Services

Burghöhe $1 \bullet 6208$ Oberkirch • Téléphone $0419250077 \bullet$ Fax 0419210586

mail@fmhconsulting.ch • www.fmhconsulting.ch 